\title{
Immediate Effect of Strain Counterstrain Technique versus Muscle Energy Technique on Upper Trapezius Tenderness in Non-Specific Neck Pain
}

\author{
Nawal M Mulla', Parag Kulkarni², Ajay Kumar ${ }^{3}$ \\ ${ }^{1}$ M.P.T, DPO's NETT College of Physiotherapy, Thane, Maharashtra, India. \\ ${ }^{2}$ M.P.Th, Assistant Professor, DPO's NETT College of Physiotherapy, Thane. \\ ${ }^{3}$ Principal, DPO's NETT College of Physiotherapy, Thane. \\ Corresponding Author: Nawal M Mulla
}

\begin{abstract}
Background: The most common form of neck pain is non-specific which is deep, dull aching $\&$ poorly localized. Non-specific pain is associated with upper trapezius tenderness. For people who work at desks and computers, or who spend many hours driving, have poor ergonomics which causes the upper trapezius to become sore and painful. The soreness can lead to the development of tender points which can be the major reason for developing neck pain and muscle spasm. Physiotherapy techniques like muscle energy technique and Strain counterstrain has been proposed to treat tenderness in trapezius.

Objective: To compare the immediate effect of Strain Counterstrain Technique \& Muscle Energy Technique on pain, pain pressure sensitivity \& mobility on non-specific neck pain with upper trapezius tenderness using Visual analogue scale, Pressure Algometer \& Universal Goniometer respectively.

Method: 60 subjects were selected as per inclusion and exclusion criteria\& were randomly assigned to Group A $(n=30)$ \& Group B $(n=30)$; Strain Counterstrain and Muscle Energy Technique respectively. Visual Analogue Scale, Pain Pressure Sensitivity \& Cervical Rotation \& Lateral Flexion Motion bilaterally was noted before \& after application of both techniques and the data was statistically analysed.
\end{abstract}

Result: Both groups showed statistical significance $(p<0.05)$ differences in all outcome measures between pre test and post test values of Group A and Group B but there is no statistical significant $(p>0.05)$ difference in the outcome measures between groups.

Conclusion: The study concludes that both techniques are equally effective in treating pain, increasing lateral flexion and rotation range of motion, and improving pain pressure sensitivity.

Keywords: Strain Counterstrain, muscle energy technique, upper trapezius tenderness, visual analog scale, non-specific neck pain, pain pressures sensitivity

\section{INTRODUCTION}

Neck pain is a condition that places a large economic burden on the health care system. In majority of the cases, the pathoanatomical source of an individual's pain cannot be identified and is therefore termed as non-specific. ${ }^{[1]}$ Symptoms vary with physical activity and change with time. Each form of acute, sub-acute or chronic neck pain, where no abnormal anatomic structure can be identified as a source of pain is called non-specific neck pain. Nonspecific neck pain is the commonest cause of neck symptoms \& results from postural \& mechanical causes. Non-specific neck pain is diagnosed on clinical grounds alone provided there are no red flags suggesting any serious condition. The main symptoms being pain in the cervical region radiating to the occiput, shoulders \& upper limbs. [2] Non-specific neck pain is often associated with upper trapezius tenderness. Typically the area of pain involved with trapezius 
being superior fibres. There has been found that the prevalence of non-specific neck pain with upper trapezius tenderness to be around $13 \%-19 \%$ in general population. ${ }^{[3]}$ For people who work at desks and computers, or who spend many hours driving, have poor ergonomics which causes the upper trapezius muscle to become very sore and painful. The soreness can lead to the development of tender points which can be the major reason for developing neck pain and muscle spasm. ${ }^{[4]}$ Many studies have hypothesized that the probable cause of tenderness pathogenesis results from the overloading and injury of muscle tissue which leads to involuntary shortening of localized fibers. The area where the soft tissue is stressed receives less oxygen, glucose, and nutrient delivery, and subsequently accumulates high levels of metabolic waste products. The result of this cascade of events is the creation of altered tissue status, pain, and the development of tender points. ${ }^{[1]}$ Tender points (TePs) are defined as small, dense and hypersensitive points found within the subcutaneous, muscular or fascial tissues. Tender points and myofascial trigger points are usually associated in the literature, although the two disorders have different features. A tender point is a localized area of tenderness in a muscle, muscle tendon junction, fat pad, or bursa region. ${ }^{[5]}$ TePs have been associated with hyperalgesia and therefore limited range of motion (ROM) and are clinically important to identify as these possess the potential to restrict functional activities. Identification is accomplished through the recognition of a pattern of clinical signs on physical examination. Signs may include the presence of a tender spot within the muscle, the presence of restricted ROM of the affected tissues. When local pressure applied to a tender area produces local pain without referred pain or pain reproduction, this area is properly considered a tender rather than a trigger point. ${ }^{[6]}$

Strain-counterstrain (SCS), also known as Positional Release Technique, is an indirect osteopathic technique, whereby dysfunctional joints and their muscles are moved away from their restrictive barriers into positions of ease in the treatment of musculoskeletal dysfunctions. The application of SCS technique requires a practitioner to first palpate a tender point (TeP) in the soft tissues, the patient's limb is then moved in such a way that the pain associated with pressure on the TeP is relieved by at least 70 percent to find the position of ease. ${ }^{[7][8][9]}$ It is theorized that the shortening or "folding-over" of aberrant tissues in SCS technique achieves its therapeutic modifications via both proprioceptive (Korr, 1975) and nociceptive mechanisms (Bailey \& Dick, 1992). [8] Bailey \& Dick (1992) proposed a hypothesis that tissue damage in dysfunctional muscles can be reduced by the positional release mechanism utilized by SCS. They suggest that relaxation of the damaged tissues may be achieved by placing patients in a position of ease which may advance local perfusion of fluids (i.e. blood, and lymph) and enhance the removal of sensitizing inflammatory mediators. ${ }^{[10]}$

Muscle Energy Technique (MET) incorporates precisely directed and controlled, patient initiated, isometric and/or isotonic contractions, designed to improve musculoskeletal function and reduce pain. It is an active muscle based treatment approach that involves the voluntary contraction of a subject's muscle in a precisely controlled direction, against a counterforce provided by the therapist. The MET may be used to decrease pain, stretch tight muscles and fascia, reduce muscle tone, improve local circulation, strengthen weak musculature and mobilize joint restrictions .Post isometric relaxation (PIR) is a division of MET used to relax and lengthen a hypertonic and shortened muscle .Post Isometric Relaxation (PIR) is the effect of the decrease in muscle tone in a single or group of muscles, after a brief period of submaximal isometric contraction of the same muscle. PIR works on the concept of autogenic inhibition. Autogenic inhibition occurs during PIR which causes 
muscle inhibition during contraction due to the activation of the Golgi tendon organ (GTO) and the muscle spindles. ${ }^{[11]}$

\section{MATERIALS AND METHODS}

Ethical committee approval was obtained. 60 subjects(both genders) in the age group of 20-30 years with non-specific neck pain participated in this study \& were recruited through Purposive Sampling \& were randomly assigned to either Group A $(n=30)$ which received Strain Counter strain Technique or to Group B $(n=30)$ which received Muscle Energy Technique. The criteria for inclusion in this study was Nonspecific neck pain of less than 3 weeks duration, unilaterally pain over upper trapezius of more than 5 on Visual Analogue Scale \& Limitation of lateral flexion or rotation of neck due to pain. Subjects who had Signs of Recent surgery, whiplash injury or open wounds to neck, Cervical spine pathologies like radiculopathies, Sensory changes in the trapezius region, Deformities like torticollis, Taking medications for pain or Vascular syndromes were excluded.

\section{Procedure:}

A written consent was taken from all the subjects in the language best understood by them. Selection of the subjects was done as per the inclusion and exclusion criteria. Purpose of the study and procedure was explained to the subjects prior to the study. Demographic data was noted down. Prevalues of Active Range of Lateral Flexion and rotation of cervical spine bilaterally using universal goniometer, pain intensity on VAS \& PPT was checked by pressure algometer before treatment.

\section{METHOD}

Patient position: Supine position.

Group A was given strain counterstrain. The subject lies supine with therapist standing on the affected side; tender points are located along the upper fibres of the trapezius. The most dominant tender point was determined by pincer palpation and marked with a small dot on the skin. Subjects were encouraged to relax as much as possible. The therapist applied gradually increasing pressure by thumb over the tender point of upper trapezius muscle until the sensation of pressure became one of pressure and pain. . At the same time, the therapist created a new position with less tension resulting in a subjective reduction of pain of up to $70 \%$. The position that led to reduced pain was ipsilateral side flexion of the cervical spine with slightly contra lateral rotation $\left(5-8^{\circ}\right)$. The patient's upper extremity was positioned in passive abduction and maintained for 90s. Finally, the subject was slowly placed into a neutral position of the cervical spine. ${ }^{[20]}$. (Fig 1)

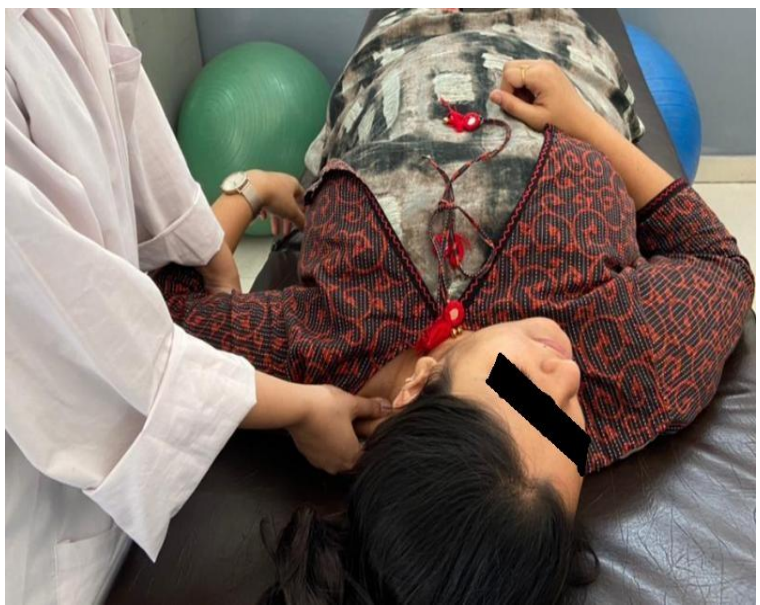

Figure 1: Strain Counterstrain for upper trapezius.

Group B was given MET. MET technique was applied with the patient in supine lying position. The researcher, with one hand on the occipital region and the other stabilizing the shoulder, performed a passive contralateral flexion to the muscle, taking the subject's head until an end-feel point without creating discomfort. At this point, subjects were asked to perform an isometric contraction of $20 \%$ (light resisted effort) of their maximum force. The subject accomplished an ipsilateral flexion of the affected muscle that is the shrugging movement caused by taking the stabilized shoulder towards the ear and the ear towards the shoulder for 7 seconds, while the researcher offered manual resistance. There should be no pain. Afterwards, the subject is relaxed and the researcher gently eases the 
neck into an increased degree and the shoulder was stretched caudally. This sequence was repeated 3 times. At the end the researcher passively guided the cervical spine into neutral position. (Fig 2)

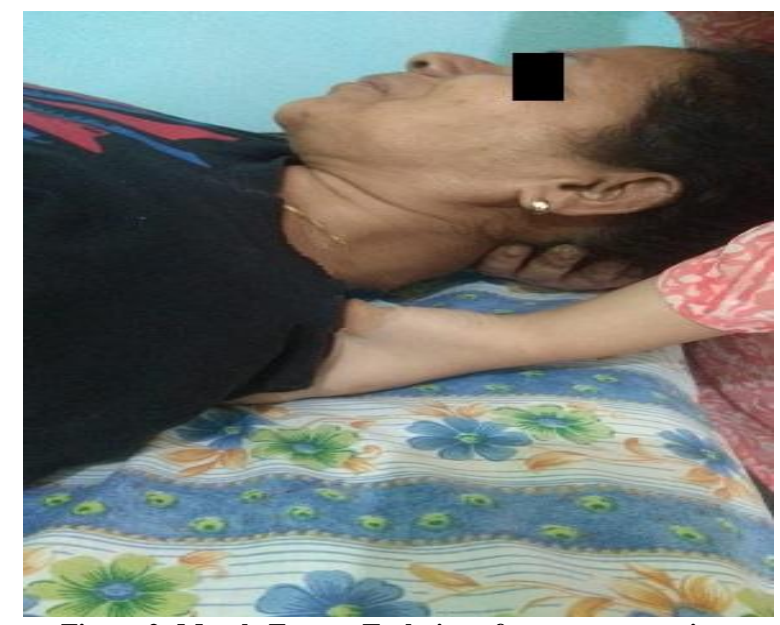

Figure 2: Muscle Energy Technique for upper trapezius.

After technique application again scores for outcome measures were noted down.

The data was collected and statistically analysed.

\section{STATISTICAL ANALYSIS:}

Collected data was entered in Microsoft Excel and SPSS software version 16 was used for the data analysis. Normality of the data was tested using the ShapiroWilk test. Since the data was not normally distributed for all the outcome measures, statistical analysis was done using the nonparametric tests. For intra group analysis the Wilcoxon test \& inter group Mann Whitney U test was used.

\section{RESULTS}

When analysed statistically using Mann Whitney test, scores of VAS\& PPT showed no statistically significant difference in pain \& pain threshold between the Group A \& Group B (p value >0.05). Also scores of AROM for Lateral Flexion \& Rotation bilaterally showed no statistically significant difference between the Group A \& Group B ( $>>0.05)$. However using Wilcoxon test intra group analysis of Group A \& Group B showed statistical significance in all outcome measures $(\mathrm{p}<0.05)$.

\begin{tabular}{|l|l|l|l|l|}
\hline $\begin{array}{l}\text { A. Comparison of Group A and Group B between the groups } \\
\text { for VAS }\end{array}$ & \multicolumn{3}{|c|}{ Values } \\
\cline { 2 - 5 } & Mean & SD & z value & $\begin{array}{l}\text { p value } \\
\text { (between group) }\end{array}$ \\
\hline Difference post-treatment Group A & 2.33 & 1.24 & & 0.64 \\
\hline Difference post-treatment Group B & 2.10 & 0.90 & -0.462 & \\
\hline
\end{tabular}

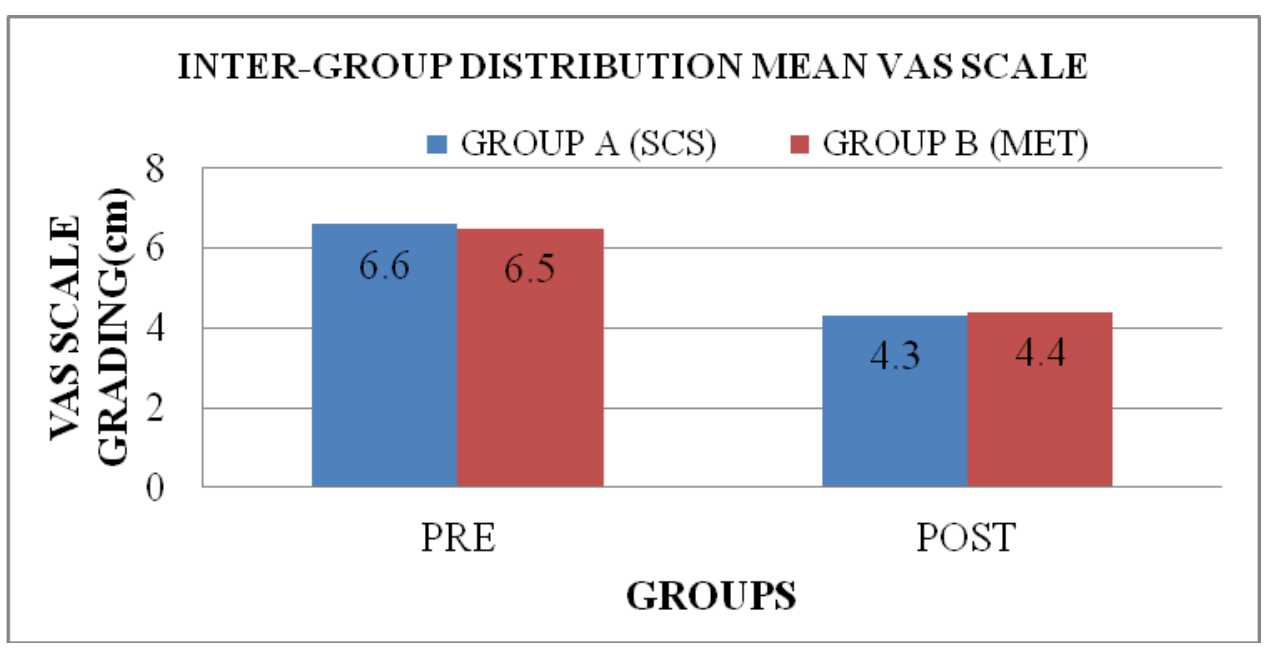

A) The above table and graph shows the mean pain scores between the groups: Group A and Group B. Inter-group comparison of mean VAS score post treatment did not show any statistically significant improvement between group A and group B ( $p$ value $>0.05$ ).

\begin{tabular}{|l|l|l|l|l|l|}
\hline \multirow{2}{*}{$\begin{array}{l}\text { B. Comparison of Group A and Group B } \\
\text { between the groups for PPT }\end{array}$} & \multicolumn{2}{|c|}{ Values } & Inference \\
\cline { 2 - 5 } & Mean & SD & z value & p value (between group) & \\
\hline Difference post-treatment Group A & 5.03333 & 2.235811 & & \multirow{2}{*}{ Non-Significant } \\
\hline Difference post-treatment Group B & 3.633333 & 2.8343136 & -1.8 & 0.06 & \\
\hline
\end{tabular}


Nawal M Mulla et.al. Immediate effect of strain counterstrain technique versus muscle energy technique on upper trapezius tenderness in non-specific neck pain.

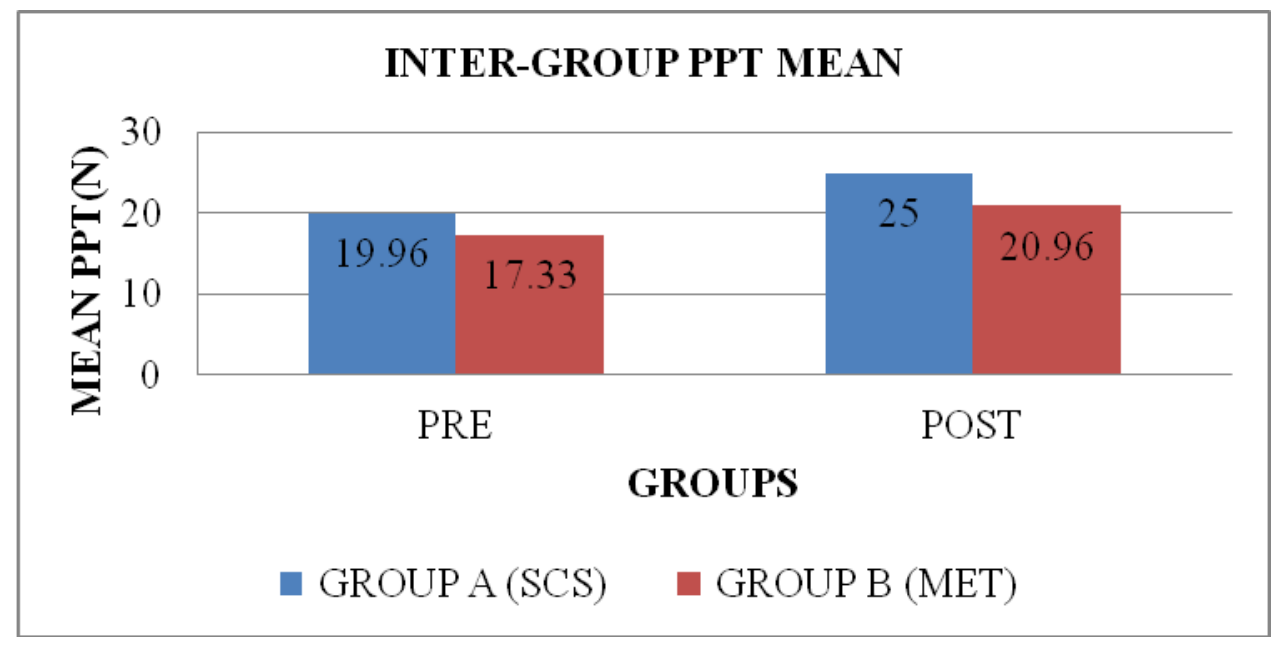

B) The above table and graph shows the mean pain pressure threshold scores between the groups: Group A and Group B. Inter-group comparison of mean PPT post treatment did not show any statistically significant improvement between group A and group B ( $p$ value $>0.05)$.

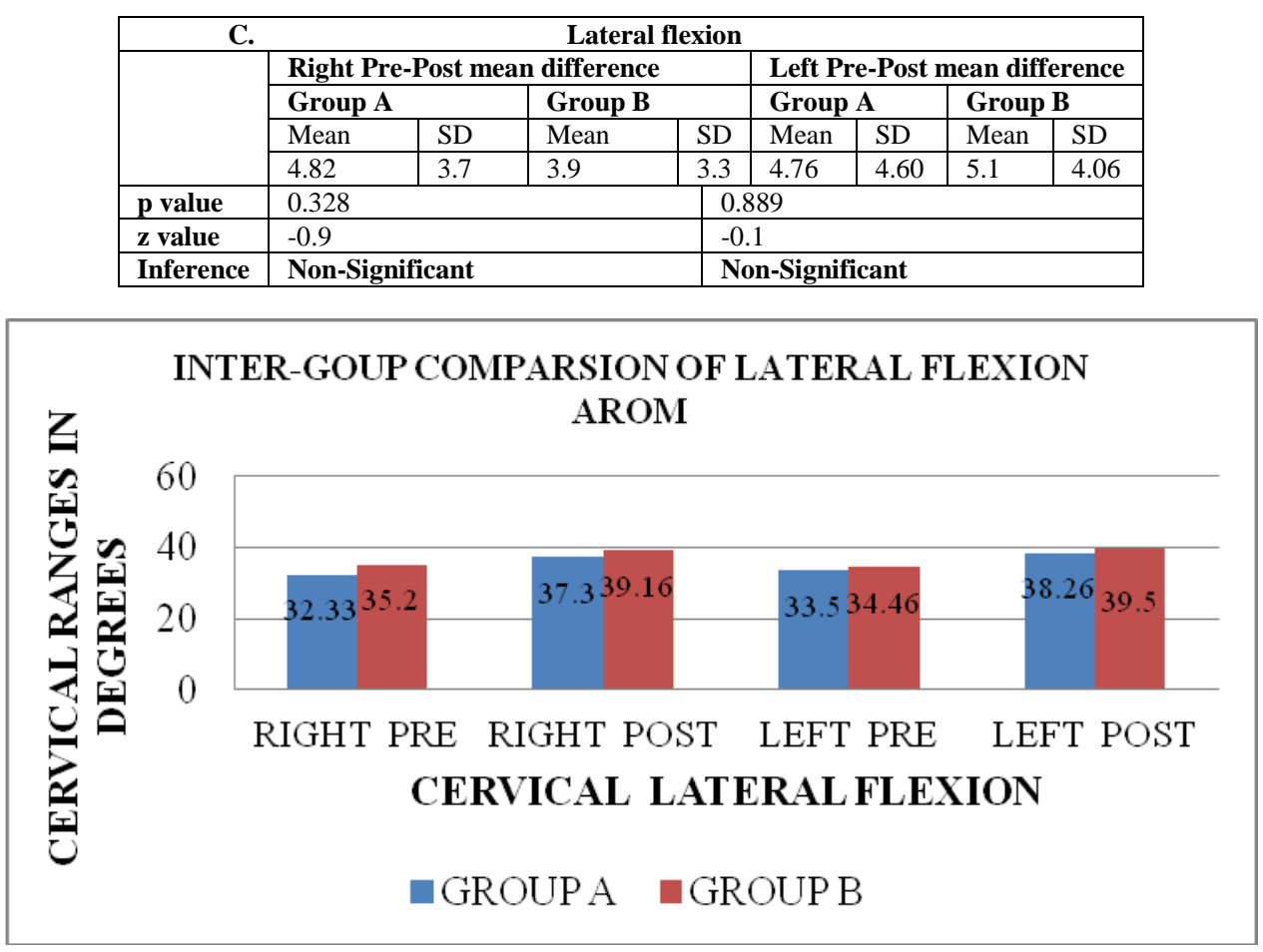

C) The above table and graph shows the mean lateral flexion range between the groups: Group A and Group B. Inter-group comparison of mean AROM of Lateral Flexion post treatment did not show any statistically significant improvement between group A and group $\mathrm{B}$ ( $\mathrm{p}$ value $>0.05$ ).

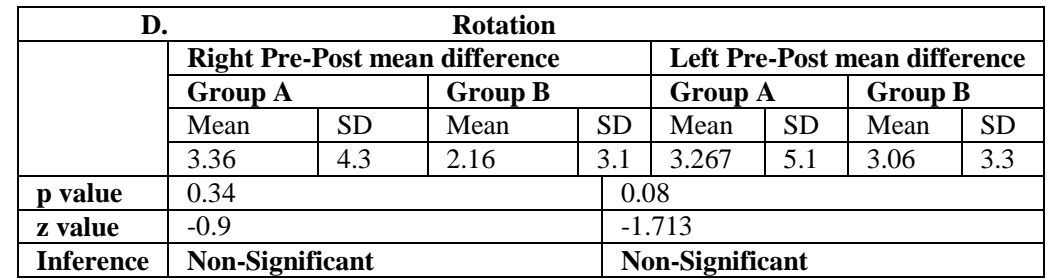




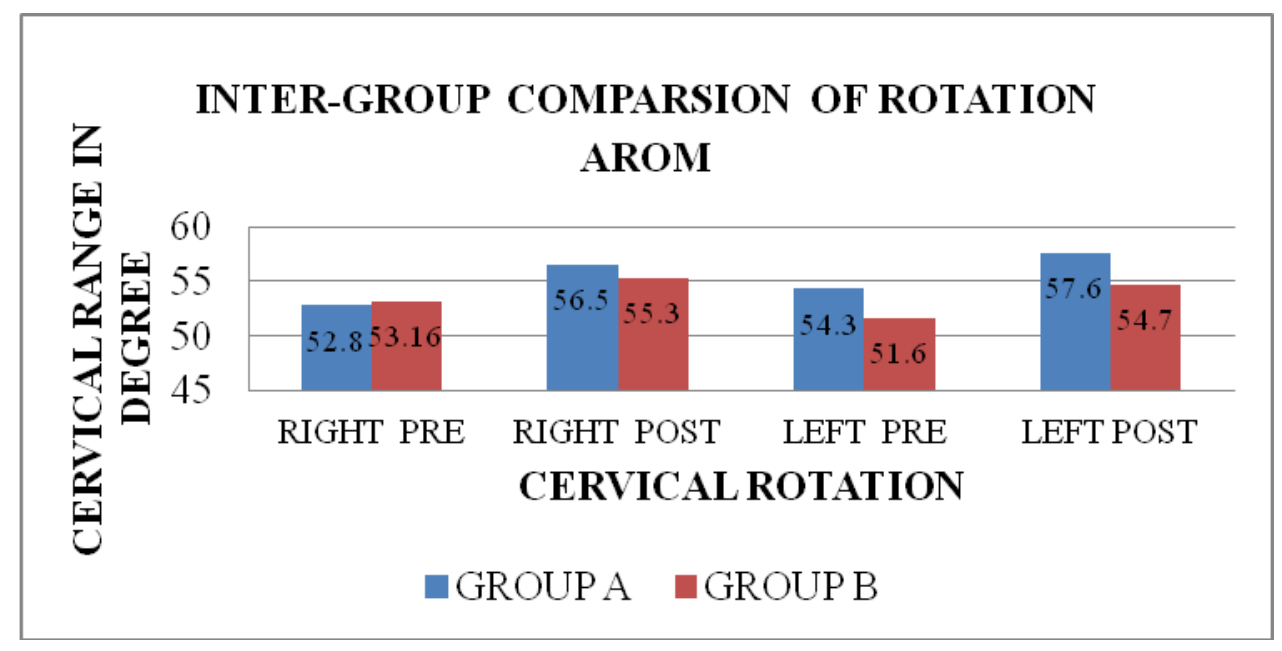

D) The above table and graph shows the mean cervical rotation range between the groups: Group A and Group B. Inter-group comparison of mean AROM of Rotation post treatment did not show any statistically significant improvement between group A and group B (p value $>0.05$ ).

\section{DISCUSSION}

The purpose of the study was to compare the immediate effects of Strain Counter Strain Technique (SCS) and Muscle energy technique (MET) on pain, pain pressure sensitivity and cervical spine mobility in upper trapezius tenderness in non specific neck pain.

60 patients who had complaints of non-specific neck pain with upper trapezius tenderness were selected for this study following random sampling method. The subjects were divided into two equal groups, 30 subjects in each group. The subjects in Group A underwent Strain-Counterstrain Technique whereas the subjects in group B received Muscle Energy Technique.

Overall results of this study showed improvements in the outcome measures immediately after both treatments. These improvements were seen in individual groups but there was no statistically significant difference in the outcome measures between the 2 groups. Therefore the null hypothesis of the study was accepted and the alternative hypothesis was rejected.
This study found the immediate effectiveness of SCS on upper trapezius tenderness to reduce pain, improve pain threshold and improve the range of motion. In Group A, effectiveness on VAS score was found. Wong and Schauer-Alvarez (2004) examined and compared the effects of SCS and SCS plus exercise on pain of the hip musculature and concluded that SCS had an immediate effect in reducing tender point pain. ${ }^{[12]}$

AROM of cervical lateral flexion \& rotation improved immediately after treatment. Strain counter-strain technique can reduce pain thereby increase the function of the neck. Dardzinski J A (2000) found a reduction of pain and an increase in neck function of $50 \%-100 \%$ in 19 of 20 patients immediately after SCS therapy. The partial improvement was maintained for six months in 11 of 20 patients, and four were still pain-free. ${ }^{[13]}$ This shows that SCS technique is effective to reduce pain and improve the range of motion.

The present study demonstrated that tender point sensitivity decreased i.e the Pain Pressure Threshold (PPT) increased in response to a single application of the strain/counterstrain technique. Meseguer et al. (2006) concluded that the application of SCS technique may be effective in producing hypoalgesia and decreased reactivity of TePs. The authors were able to demonstrate an immediate decrease in the sensitivity of a chosen TeP following the application of SCS technique. The results of 
the present study also agree with those of Wong and Schauer-Alvarez who also found that strain-counterstrain reduced sensitivity to palpation in subjects exhibiting tender points in the hip musculature (p value $=0.000$ ) Strain/counterstrain is thought to achieve its benefits by automatic resetting of muscle spindles which would help to dictate the length and tone of the affected tissues. Strain/counterstrain technique is applied with the targeted muscle in a shortened position, and used to treat tender points. It might be that pain relief from strain/counterstrain technique may result from the stimulation of $A \partial$ fibers. ${ }^{[5]}$

This study also found the effectiveness of MET on upper trapezius tenderness amongst the subjects in Group B. The subjects who received MET its overall effectiveness on VAS score was found immediately after treatment. AROM was found to improve \& PPT also improved. The results of studies with MET intervention and outcome measures such as pain on VAS, PPT and flexibility support the results of the present study, which indicates an immediate increase in the active cervical range of motion and PPT and an immediate decrease in pain on VAS. Campelo et al. (2013) assessed the effects of different manual techniques such as ischemic compression (IC), passive stretching, and MET on the cervical ranges of motion and pressure pain sensitivity in subjects with LTrPs of the upper trapezius muscle. The outcomes were assessed prior to the treatment, immediately after the treatment, 24 hours after the intervention, and one week later. All the manual interventions on the upper trapezius with LTrPs seemed to improve the cervical range of motion and the pressure pain sensitivity immediately after the treatment. ${ }^{[14]}$

Sadria G et al. (2016) compared the immediate effects of two manual treatment regimens: MET and Active Release Therapy (ART) on individuals with upper trapezius trigger points. The outcomes were pain on VAS, upper trapezius thickness and active lateral cervical flexion. The results showed a significant reduction in pain and muscle thickness and increase in lateral cervical flexion ROM; none of the techniques being superior to each other. ${ }^{[15]}$

Cassidy et al found immediate increase in ROM of neck in all three planes in patients with mechanical neck pain who were mobilized using MET. ${ }^{[16]}$

The effects of MET for increase in ROM post intervention can be explained on the basis of reflex muscle relaxation following contraction that has been proposed to occur by activation of the golgi tendon organs and their inhibitory influence on the $\alpha$-motor neuron pool. MET was found to reduce pain perception. Activation of muscle and joint mechanoreceptors leads to sympathoexcitation by somatic efferents and causes localized activation of periaqueductal gray matter that plays a role in descending modulation of pain. ${ }^{\text {[14][17][15] }}$

The objective of the study was to compare the effectiveness of SCS (Group A) versus MET (Group B) in the management of upper trapezius tenderness. Statistical analysis shows, comparing VAS score between Groups A and B, (p value $=0.64)$ no significant change in pain reduction between the groups. Statistical analysis shows same improvement in Group $A$ in cervical side flexion \& rotation as compared to Group B ( $\mathrm{p}$ value >0.05). Also there was no statistically significant difference in the improvement of PPT between the groups $(p=0.06)$. Our results are supported by the study of Ellythy et al., who conducted a study on low back dysfunction with help of MET and SCS and proved that both MET and SCS techniques are effective in reducing pain and functional disability in patients with chronic low back pain. ${ }^{[18]}$

Sabby et al., who have conducted a study on upper trapezius MTrP with help of MET and SCS also concluded an improved range of motion, reduced pain in patients with upper trapezius MtrP in both the groups. ${ }^{[19]}$

Naik Prashant P (2010). in his study "Comparison of muscle energy technique and positional release therapy in acute low 
back pain" found both MET and PRT along with HMP for acute low back pain showed improvement following 8 days of treatment as per significant decrease in pain (VAS), amount of lumbar extension ROM, and disability level as per MODQ. ${ }^{[20]}$

It was found by Bhojan Kannabiran., et al. that there was significant decrease in pain and increase in lumbar range in both the groups with muscle energy technique and positional release technique but there was no significant difference between the groups which is similar to our study. ${ }^{\text {[21] }}$

\section{Mechanism:}

SCS is believed to achieve its benefits by means of an automatic resetting of muscle spindles, which would help to dictate the length and tone into the affected tissues and increase the length of sarcomere.PPT and local pain intensity improvement after application of SCS can also be due to the manual contact component of the treatment and the stimulation of A $\partial$ fibres; a process that can lead to blockage of the pain. ${ }^{[5][22]}$

In this method, following the release of pressure on TeP, tissue blood and lymphatic circulation of that area increases which removes the hypoxic conditions in the muscle and results in cellular metabolism leading to the removal of inflammatory chemical substances such as prostaglandins, histamine, and bradykinin; therefore, reduction of sensitization of nociceptors occurs. Also, one of the advantages of SCS is breaking the cycle of pain-spasm-pain. ${ }^{[23]}$

In case of MET, its application seems to affect the viscoelastic and plastic tissue properties. MET may also influence pain mechanisms and promote hypoalgesia. Studies have suggested that MET related post-isometric techniques reduce pain and discomfort when applied to the muscles. Although the mechanisms of such an influence are not known, they may involve central and peripheral modulatory mechanisms, such as the activation of the muscle and joint mechanoreceptors that involve centrally mediated pathways, like the periaqueductal gray in the midbrain and noradrenergic descending inhibitory pathways. MET may increase fluid drainage and augment hypoalgesia. Rhythmic muscle contraction increases muscle blood and lymph flow rates, \& increases transcapillary blood flow. ${ }^{[15]}$

\section{FURTHER SCOPE OF STUDY}

Long-term follow-up should be evaluated .Study should be performed using trigger points/tender points of other muscles associated with non-specific neck pain.

Study can be performed with different treatment techniques for different age-group patients with different type's neck pain.

Study should be performed including flexion and extension of the cervical vertebra

\section{CONCLUSION}

The study concludes that Strain Counter Strain Technique \& Muscle Energy Technique are effective in treating pain, increasing lateral flexion and rotation range of motion, and improving pain pressure sensitivity.

But comparing both groups proved that there is no difference between groups on comparing pain, cervical range of motion, \& pain pressure sensitivity.

Hence both techniques are equally effective in treating patients with nonspecific neck pain with upper trapezius tenderness.

\section{Clinical Implication:}

Results suggested that both the techniques i.e. strain counterstrain technique $\&$ muscle energy technique are effective in reducing Pain and in improving Cervical Range of Motion \& Pain Pressure Sensitivity in non-specific neck pain with upper trapezius tenderness. On the initial day, SCS \& MET make an excellent approach because treatment is both gentle and effective. SCS \& MET can be performed prior to other rehabilitation 
techniques, such as strengthening exercises, to decrease pain and improve disability. Manual therapy is also found to be more cost-effective for treating nonspecific pain than physiotherapy or care by a general practitioner.

\author{
Abbreviations \\ SCS- Strain Counterstrain, MET- Muscle \\ Energy Technique, VAS- Visual Analogue \\ Scale, \\ PPT- Pain Pressure Threshold, AROM - \\ Active Range Of Motion, TePs-Tender \\ points
}

\section{ACKNOWLEDGEMENTS}

I express my deep sense of gratitude and sincere thanks to our respected sir Dr. Ajay Kumar and guide Dr. Parag Kulkarni who immensely helped me with sincere guidance, untiring cooperation, valuable advice and endless inspiration during the course of the study. I also take this opportunity to thank my family members and friends for their help and support.

\section{Conflict of Interest: None}

\section{Source of Funding: None}

\section{Ethical Approval: Approved}

\section{REFERENCES}

1. Nagrale Et Al. The Efficacy Of An Integrated Neuromuscular Inhibition Technique On Non-Specific Neck Pain. Journal Of Manual And Manipulative Therapy 2010; 18(1)

2. Binder.The Diagnosis \& Treatment Of NonSpecific Neck Pain \& Whiplash.Eura Medicophys.2007;43:79-89

3. Andersen Et Al.: Prevalence And Anatomical Location Of Muscle Tenderness In Adults With Nonspecific Neck/Shoulder Pain. BMC Musculoskeletal Disorders 2011 12:169.

4. Ravish $\mathrm{v}$ n,Helen s.To compare the effectiveness of myofascial release technique versus positional release technique With Laser In Patients With Unilateral Trapezitis.Journal Of Evolution
Ofmedical And Dental Sciences. 2014 Mar; 3(9):2161-7.

5. A.A. Meseguer Et Al. Immediate Effects Of The Strain/Counterstrain Technique In Local Pain Evoked By Tender Points In The Upper Trapezius Muscle Clinical Chiropractic (2006) 9, 112-118

6. Borg-Stein \& Stein Trigger Points And Tender Points One And The Same? Does Injection Treatment Help? Rheumatic Disease Clinics Of North America 1996 May; Volume 22 Number 2.

7. D’Armbrogio, K.J., Roth, G.B. Positional Release Therapy: Assessment \& Treatment Of Musculoskeletal Dysfunction. St. Louis: Mosby; 1997. Pp. 20.

8. Jones, L. H. Strain And Counterstrain. Indianapolis, USA: The American Academy Of Osteopathy ; 1981.

9. Ward, R. . Foundations For Osteopathic Medicine, 2 Ed. Philadelphia, USA: Lippincott Williams \& Wilkins; 2003.

10. Bailey, M., \& Dick, L. Nociceptive Considerations In Treating With Counterstrain. J Am Osteopath Assoc, 1992; 92(3), 334-341.

11. Chaitow L. Muscle Energy Techniques. : Elsevier Health Sciences; 2013.

12. Wong CK, Schauer-Alvarez C. Effect of strain/counterstrain on pain and strength in hip musculature. J Man Manip Ther 2004; 12:215-23.

13. Dardzinski JA, Ostrov BE, Hamann LS. Myofascial pain unresponsive to standard treatment: successful use of a strain and counterstrain technique with physical therapy. J Clin Rheumatol. 2000; 6(4):169174.

14. Natália M. Oliveira-Campelo, Cristina A. De Melo, Francisco Alburquerque-Sendín And Jorge P. Machado Short- And MediumTerm Effects Of Manual Therapy On Cervical Active Range Of Motion And Pressure Pain Sensitivity In Latent Myofascial Pain Of The Upper Trapezius Muscle:A Randomized Controlled Trial Journal Of Manipulative And Physiological Therapeutics Manual Therapy, 2013.

15. Sadria, G., Hosseini, M., Rezasoltani, A., Akbarzadeh Bagheban, A., Davari, A., \& Seifolahi, A. A Comparison Of The Effect Of The Active Release And Muscle Energy Techniques On The Latent Trigger Points Of The Upper Trapezius. Journal Of 
Nawal M Mulla et.al. Immediate effect of strain counterstrain technique versus muscle energy technique on upper trapezius tenderness in non-specific neck pain.

Bodywork And Movement Therapies, 2017; 21(4), 920-925.

16. Thomas E, Cavallaro AR, Mani D, Bianco A, Palma A. The efficacy of muscle energy techniques in symptomatic and asymptomatic subjects: a systematic review. Chiropr Man Therap. 2019; 27:35.

17. El-Laithy MH And Fouda KZ. Effect Of Post Isometric Relaxation Technique In The Treatment Of Mechanical Neck Pain. Phys Ther Rehabil. 2018; 5:20.

18. Marzouk A. Ellythy Efficacy Of Muscle Energy Technique Versus Strain Counter Strain On Low Back Dysfunction Bull. Fac. Ph. Th. Cairo Univ., 2012, Jul; Vol. 17, No. (2).

19. Nambi GS, Sharma R, Inbasekaran D, Vaghesiya A, Bhatt U. Difference in effect between ischemic compression and muscle energy technique on upper trepezius myofascial trigger points: Comparative study. Int J Health Allied Sci 2013; 2:17-22.

20. Naik Prashant P., Heggannavar Anand, Khatri Subhash M. Comparison of muscle energy technique and positional release therapy in acute low back pain - RCT.
Indian Journal of Physiotherapy and Occupational Therapy 2010; 4(2): 32.

21. Bhojan Kannabiran., et al. "A Comparative Study of the Effectiveness of Two Manual Therapy Techniques on Pain and Lumbar Range of Motion in Individuals with Mechanical Low Back Ache". EC Orthopaedics, 2015, 2.1: 36-42.

22. Leon chaitow. Positional release techniques, 3 ed.; 2007.

23. M. Mohammadi Kojidi , F. Okhovatian, A. Rahimi,A.A. Baghban , H. Azimi The Influence Of Positional Release Therapy On The Myofascial Trigger Points of The Upper Trapezius Muscle In Computer Users Journal Of Bodywork And Movement Therapies , 2016, Oct ; Volume 20, Issue 4, Pages 767-773.

How to cite this article: Mulla NM, Kulkarni P, Kumar A. Immediate effect of strain counterstrain technique versus muscle energy technique on upper trapezius tenderness in nonspecific neck pain. International Journal of Science \& Healthcare Research. 2021; 6(2): 289-298. DOI: https://doi.org/10.52403/ijshr. 20210452 